\title{
Québec ZéN : une initiative de la société civile pour coconstruire le Québec carboneutre de demain
}

\section{Carole Dupuis}

\section{(2) OpenEdition \\ 1 Journals}

Édition électronique

URL : https://journals.openedition.org/ere/8393

ISSN : 2561-2271

\section{Éditeur}

Centr'ERE

\section{Référence électronique}

Carole Dupuis, «Québec ZéN : une initiative de la société civile pour coconstruire le Québec carboneutre de demain », Éducation relative à l'environnement [En ligne], Volume 17-1 | 2022, mis en ligne le 27 janvier 2022, consulté le 03 février 2022. URL : http://journals.openedition.org/ere/8393

Ce document a été généré automatiquement le 3 février 2022.

La revue Éducation relative à l'environnement est mise à disposition selon les termes de la Licence Creative Commons Attribution - Pas d'Utilisation Commerciale 4.0 International. 


\title{
Québec ZéN : une initiative de la société civile pour coconstruire le Québec carboneutre de demain
}

\author{
Carole Dupuis
}

1 En septembre 2019, le Front commun pour la transition énergétique donnait le coup d'envoi public de «Québec ZéN » (zéro émission nette), une initiative de la société civile visant la transition du Québec vers la carboneutralité dans un temps opportun et un esprit de justice sociale. En soutien à cette démarche, le Front commun publiait le même jour la version 1.0 de sa Feuille de route pour la transition du Québec vers la carboneutralité. Fruit d'un intense travail collaboratif, ce document était proposé en tant qu'ancrage aux échanges approfondis auxquels le Front commun conviait l'ensemble de la société québécoise.

2 Deux ans plus tard, le projet Québec ZéN a pris une ampleur inattendue. L'engouement que l'initiative suscite invite à prendre un pas de recul pour la présenter, réfléchir à la théorie du changement qui lui est sous-jacente et commencer à en tirer certains constats.

\section{Le Front commun pour la transition énergétique}

Le Front commun pour la transition énergétique ${ }^{1}$ est né en 2015 de la volonté d'une trentaine de groupes citoyens et d'organisations environnementales de se concerter afin de combattre plus efficacement les projets d'exploration, d'exploitation et de transport des énergies fossiles au Québec. Six ans et plusieurs luttes victorieuses plus tard, le Front commun regroupe près d'une centaine d'organisations représentant collectivement 1,8 million de personnes. Beaucoup plus diversifié, son membership est issu aujourd'hui de cinq secteurs différents de la société civile: organisations environnementales, groupes citoyens, syndicats, organismes d'action communautaires et étudiants. 
4 En septembre 2017, le Front commun s'est positionné de manière distinctive en prenant position pour une transition socio-écologique qui déborde largement du faisceau habituel des préoccupations environnementales: il s'est donné comme visée une transformation systémique ancrée dans des valeurs d'inclusion et d'équité ${ }^{2}$. En mai 2018, le Front commun a affirmé sa volonté de dépasser son rôle d'organisme de concertation pour s'imposer en tant qu'acteur incontournable dans la transition juste vers une société neutre en carbone ${ }^{3}$. Cette vision s'est confirmée lors de l'approbation du projet Québec ZéN par l'assemblée générale en 2019. Elle s'est cristallisée au cours de la phase 1 de ce projet, soit la coconstruction de la Feuille de route pour la transition du Québec vers la carboneutralité, adoptée à l'unanimité par l'assemblée générale en octobre $2020^{4}$. Finalement, elle a commencé à se concrétiser lors du démarrage de la phase $2 \mathrm{de}$ Québec ZéN, qui marque le passage à l'action sur le terrain. Le projet «Collectivités ZéN », première initiative de cette phase, a été lancé publiquement en mai 2021.

\section{Le projet Québec ZéN}

5 Le projet Québec ZéN a émergé dans un contexte d'abondance des connaissances scientifiques sur le réchauffement climatique, sur ses conséquences et sur les manières de l'éviter ${ }^{5}$. D'importantes recherches multidisciplinaires sur des aspects transversaux de la crise climatique et de la transition étaient également disponibles ${ }^{6}$ et les outils de vulgarisation de ces savoirs se multipliaient ${ }^{7}$. Les environnementalistes ne cessaient d'alerter la population, de diffuser des conseils et de bombarder les gouvernements de revendications ${ }^{8}$. Pourtant, en matière d'action climatique, les progrès marquants se faisaient désespérément attendre.

6 La question était donc: Pourquoi ? Connaissant les sources des émissions de GES d'origine anthropique, sachant (au moins en grande partie) comment les éviter, consciente des risques extrêmes qu'elles représentent pour la qualité de vie des générations montantes et même la vie sur terre, pourquoi notre société n'arrive-t-elle pas à poser les gestes qui s'imposent?

7 C'est en puisant dans les expériences de vie personnelle et militante de ses membres, nourries par leurs savoirs professionnels et académiques, que le Front commun a tenté de cerner les sources de la paralysie climatique générale. La réflexion collective a mené à l'identification de quatre besoins non comblés dont la satisfaction était perçue comme étant essentielle à une démarche efficace de transition juste vers la carboneutralité. C'est à partir de ces besoins qu'ont été formulées les hypothèses de travail du projet Québec ZéN et qu'a été clarifiée la théorie du changement qui le sous-tend. Ils sont présentés de manière schématique dans la figure 1 , avec les stratégies qui en découlent et les activités conçues pour y répondre. 


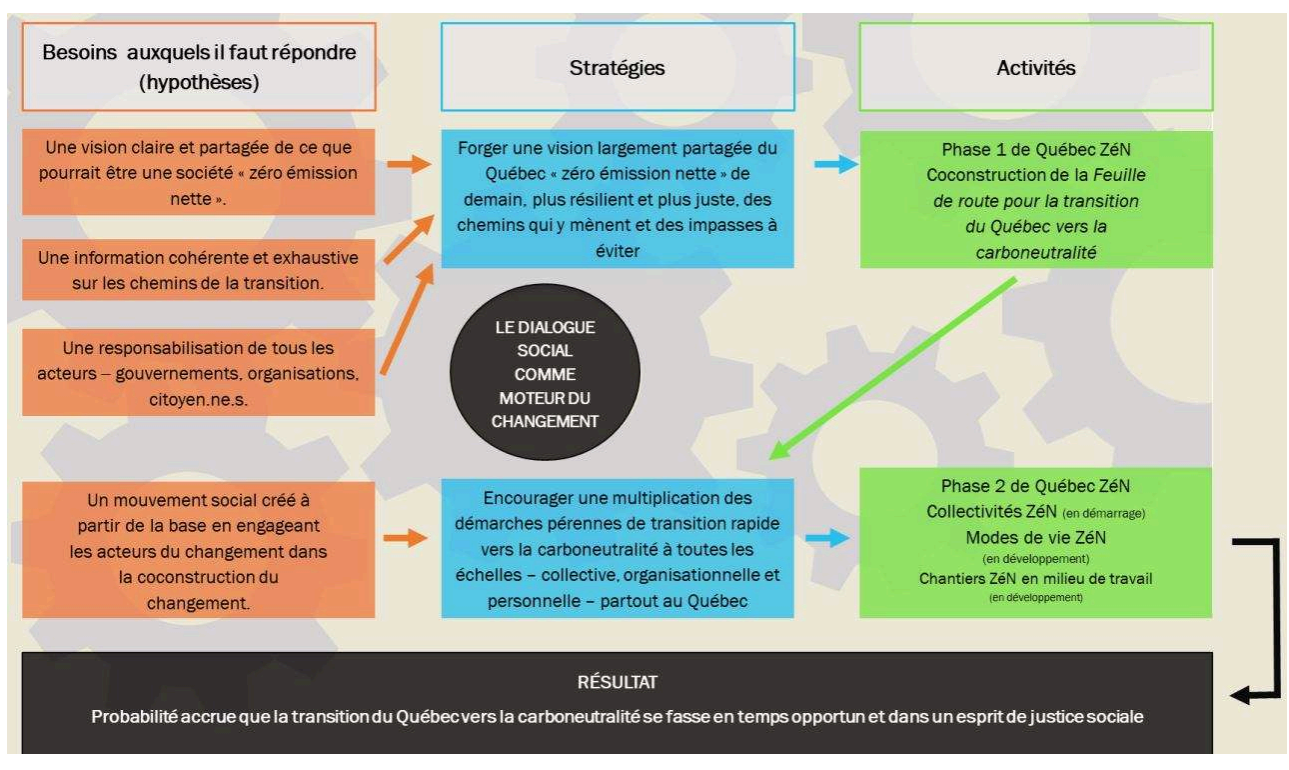

Figure 1 : Théorie du changement du projet Québec ZéN

\section{Les besoins auxquels le projet Québec ZéN ambitionne de répondre}

On peut identifier quatre principaux besoins que Québec ZéN souhaite combler pour favoriser la transformation collective qui s'impose.

\section{Une vision claire et partagée d'une société « zéro émission nette »}

Le projet Québec ZéN pose comme première hypothèse que pour se mettre en route et avancer avec confiance, une société qui a un virage important à faire - dans n'importe quel domaine - doit avoir une vision claire et partagée de la destination: il est impossible de s'engager dans un changement quand on ignore où celui-ci nous mène. Dans le cas précis qui nous occupe, on ne peut pas s'attendre à ce que la population ou les décideurs entreprennent de s'affranchir du pétrole et du gaz s'ils ne peuvent pas visualiser un Québec fonctionnant sans énergies fossiles. Cette hypothèse rejoint la théorie de Thomas Samuel Khun (1970), père du concept de "Paradigm Shift », qui a écrit : « Un paradigme n'est pas rejeté dès qu'il est réfuté, mais seulement quand il peut être remplacé. " ${ }^{9}$ Le souci de répondre à ce besoin a été le premier déclencheur du projet Québec ZéN et a dicté l'approche de coconstruction utilisée pour produire la Feuille de route pour la transition du Québec vers la carboneutralité - une approche signature du projet dans son ensemble et qui sera systématiquement reprise dans tous ses volets.

\section{Une information cohérente et exhaustive sur les chemins de la transition}

Selon la deuxième hypothèse à la base de Québec ZéN, une démarche complexe et exigeante de transformation collective ne peut être menée de manière efficace et cohérente que si les chemins menant à la situation visée sont connus et balisés. Privés de vue d'ensemble et d'information suffisante, ou gavés d'un excès d'informations à la pièce, superficielles, souvent contradictoires et parfois trompeuses sur les mesures à prendre pour juguler la crise climatique, les leaders politiques et la population ont du mal à échapper à l'incertitude paralysante. Le caractère fragmentaire de la plupart des 
«solutions » proposées alimente le scepticisme quant aux chances de réussite de la transition écologique.

Suivant cette logique, il fallait donc que le projet Québec ZéN permette de développer une représentation commune et globale des chemins à suivre pour concrétiser la société post carbone désirée, et qu'il procure une vue d'ensemble couvrant non seulement les dimensions matérielles, mais aussi l'ensemble des enjeux sociaux, politiques et économiques de la transformation à opérer. Une telle ambition exigeait la participation d'acteurs d'horizons très diversifiés afin de faire jaillir du maillage des savoirs et du choc des perspectives une vision aussi juste et consensuelle que possible.

\section{Une responsabilisation de tous les acteurs}

12 Selon l'analyse du Front commun, la dynamique d'opposition entre «les gouvernements irresponsables ", « les municipalités qui disposent de nombreux leviers pour lutter contre le réchauffement planétaire », « les compagnies sans scrupules » et «les citoyen.ne.s qui ne font pas leurs devoirs" nourrit la tendance naturelle des acteurs à se renvoyer la balle plutôt qu'à s'approprier le dossier et à prendre la situation en main, chacun à son échelle : chaque acteur se sent impuissant et alimente le sentiment d'impuissance de l'autre. Sans dédouaner les gouvernements qui n'assument pas correctement leur responsabilité de fiduciaires du bien commun, il est donc apparu nécessaire que le projet Québec ZéN mette en exergue les rôles complémentaires et indispensables que tous et chacun ont à jouer dans le succès de la transition.

\section{Un mouvement social créé à partir de la base en engageant les acteurs du changement dans la coconstruction du changement}

Enfin, la quatrième hypothèse fondatrice du projet Québec ZéN est que les gouvernements n'initieront pas la transformation profonde du système économique sans laquelle les cibles de réduction des GES ne pourront ni être atteintes en temps opportun, ni être poursuivies dans un esprit de justice sociale. Pour que l'objectif ultime du projet se réalise, il est donc impératif de faire émerger un mouvement social puissant fondé sur une appropriation du pouvoir citoyen.

\section{Le dialogue social comme moteur du changement}

On aura noté que le cadre d'analyse sous-jacent au projet Québec ZéN est plus proche $\mathrm{du}$ spectre de l'innovation sociale que de celui d'une analyse politique qui aborderait la problématique directement sous l'angle des rapports de pouvoirs. En effet, sans renoncer à transformer les rapports de pouvoir, bien au contraire, le projet Québec ZéN ne cherche pas à le faire par la revendication, la confrontation ou les actions dérangeantes, mais plutôt par le dialogue social considéré comme étant le moteur du changement. Il mise sur le plein potentiel des communautés ${ }^{10}$ concernées en alliant recherche, créativité, expérimentation et pensée critique, ainsi que concertation et cocréation entre toutes les parties prenantes. Cette posture n'en est pas une de rejet des autres théories du changement, mais plutôt de reconnaissance qu'il ne faut pas seulement refuser le statu quo, mais aussi construire l'avenir, et que la cohabitation de 
plusieurs approches différentes sera sans doute nécessaire pour induire les grands changements qui s'imposent.

\section{Phase 1 de Québec ZéN : Coconstruction de la Feuille de route pour la transition du Québec vers la carboneutralité}

Première phase du projet Québec ZéN, la Feuille de route pour la transition du Québec vers la carboneutralité s'est construite en trois itérations mettant à contribution des cercles toujours plus larges de collaborateurs. Sa version bêta incorporait déjà les commentaires d'une vingtaine de personnes, soit des militant.e.s consulté.e.s pour tester la résonance d'une telle démarche et des spécialistes interpellé.e.s pour assurer la robustesse du contenu. Présentée à l'assemblée générale en juin 2019 comme document de travail, elle s'est enrichie au cours des quatre mois suivants par les apports d'un grand nombre de membres du Front commun. Leurs contributions ont mené à la version 1.0 lancée en septembre de la même année en tant que support à une concertation encore plus vaste.

Cet appel au dialogue a entrainé une démarche collective exceptionnelle pour aboutir à la version 2.0 adoptée à l'automne 2020. Au total, près de 200 personnes issues de 85 organisations et 12 universités se sont portées volontaires pour participer à un ou plusieurs des 14 Groupes thématiques chargés d'enrichir le contenu des 14 chapitres de la Feuille de route. Les Groupes thématiques ont fonctionné en mode autogestion, avec le soutien sporadique d'une chargée de projet. Loin de s'essouffler au fil des mois, leurs travaux ont suscité un intérêt croissant et le Front commun a conséquemment reporté à l'automne 2020 la diffusion de la version enrichie de la Feuille de route, initialement prévue dans le cadre des activités du Jour de la Terre, le 22 avril 2020.

La Feuille de route vise non seulement à proposer une vision du Québec carboneutre de demain et à présenter une synthèse des savoirs scientifiques et techniques indispensables à l'évitement des émissions de GES d'origine anthropique, mais aussi à cibler les racines profondes de la crise écologique et climatique, et à tracer les contours des conditions de planification et de déploiement qui rendront socialement possible la nécessaire transformation. En plus d'indiquer des balises et de signaler les impasses à éviter, chacun de ses chapitres propose des actions concrètes aux gouvernements, aux municipalités, aux organisations, aux citoyennes et aux citoyens. La figure 2 donne une vue d'ensemble des chapitres de la Feuille de route, correspondant aux multiples dimensions à prendre en compte pour opérer une transition efficace et porteuse de justice sociale. Elle montre aussi l'interdépendance de toutes ces dimensions. 


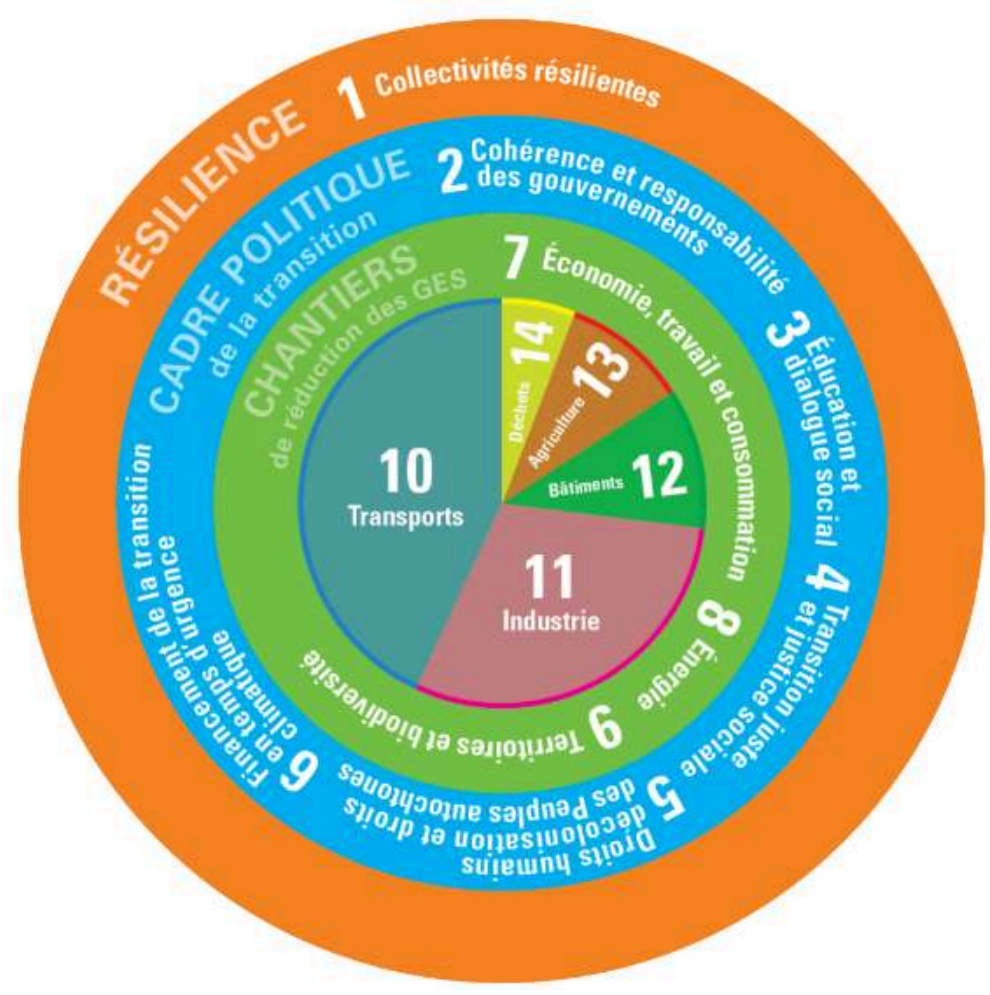

Figure 2 : Les 14 chapitres de la Feuille de route pour la transition du Québec vers la carboneutralité

\section{Phase 2 de Québec ZéN : En action !}

Intitulée «En action!», la seconde phase de Québec ZéN se concentre principalement sur l'action collective, clé de voûte des nécessaires transformations systémiques. Le Front commun reconnait toutefois que la décarbonation implique que les acteurs se mobilisent à toutes les échelles: collective, organisationnelle et personnelle. En d'autres termes, pour qu'un territoire devienne "zéro émission nette ", il faut certes que la collectivité entreprenne une démarche concertée et structurée de transition vers la carboneutralité, mais aussi que toutes les organisations (industries, commerces, institutions etc.) et tous les ménages présents sur le territoire fassent de même. Trois initiatives ciblant ces trois échelles sont présentement en démarrage ou en développement.

\section{«Collectivités ZéN » (en démarrage)}

Le projet Collectivités ZéN a pour but de puiser dans les orientations établies au cours de la phase 1 de Québec ZéN pour soutenir la coconstruction de collectivités «zéro émission nette ", plus résilientes et plus justes dans les diverses régions du Québec, selon un modèle d'engagement distribué :

- en s'appuyant sur des organismes porteurs bien ancrés dans leur milieu pour mettre en place des Chantiers de transition rassemblant des acteurs clés de tous les secteurs;

- en soutenant ces Chantiers de façon proactive, avec l'aide de partenaires nationaux et de scientifiques; 
- en adoptant une optique de développement des capacités de transformation des collectivités elles-mêmes par la coformation et le croisement des savoirs (et non en cherchant à développer et imposer un modèle unique) ;

- en engageant des cercles toujours plus larges de la société dans les collectivités visées et un nombre toujours croissant de collectivités, afin de rejoindre graduellement l'ensemble du Québec.

20 L'initiative Collectivités ZéN a été retenue en priorité pour la phase « En action! » du projet Québec ZéN car une approche territoriale brise les silos sectoriels et permet d'adopter la perspective globale nécessaire aux changements structuraux qui sont requis. Elle se prête à un exercice approfondi de dialogue social autour de la transformation d'un territoire dont les caractéristiques physiques, les infrastructures, le tissu social, la structure économique, les relations de pouvoir et les normes sociales peuvent être appréhendés par la population qui y vit.

L'originalité de Collectivités ZéN tient au rassemblement des énergies et à la mutualisation des savoirs et des acquis d'acteurs socio-économiques nationaux et locaux qui ne s'étaient pas encore unis pour relever le défi de la transition, mais qui possèdent des expertises et des réseaux complémentaires, tous essentiels au succès de l'action climatique et de la résilience. Cette initiative fait du Front commun le pivot de l'unique projet collectif de transformation systémique, opérationnalisable sur le terrain, en voie de déploiement à l'échelle du Québec.

Les quatre premiers Chantiers de Collectivités ZéN ont été annoncés le 12 mai 2021 : ClimAction Lachine, Laval ZéN, Le Grand dialogue régional pour la transition socioécologique du Saguenay-Lac-Saint-Jean et le Chantier ZéN de la région de Québec. Bien que naissantes, ces initiatives d'innovation sociale ancrées dans l'inclusion, le partage, la créativité et la solidarité créent déjà une effervescence palpable. Il est prévu que quatre nouveaux Chantiers seront lancés chaque année au cours des cinq prochaines années.

\section{« Modes de vie ZéN » (en développement)}

23 Les initiatives visant l'action climatique à l'échelle personnelle ont souvent mauvaise presse dans les milieux environnementaux, car elles tendent à faire porter le fardeau de la crise climatique aux individus alors qu'il revient d'abord aux gouvernements de créer un environnement législatif, économique et matériel menant à l'atteinte des cibles de carboneutralité. Il n'en reste pas moins indispensable que la population adhère à l'objectif, accepte les mesures de transition juste mises de l'avant par les décideurs publics et s'engage, elle aussi, dans une transformation de ses modes de vie.

Or, selon le Baromètre de l'action climatique (Champagne St-Arnaud, V. et coll., 2020) :

plus des trois quarts des Québécois et des Québécoises veulent en faire davantage pour le climat ( $78 \%)$, mais la majorité continue de confondre enjeux climatiques et autres enjeux environnementaux, ce qui crée un écart entre les intentions et les comportements réels en matière d'action climatique. En effet, malgré leurs bonnes intentions, les citoyens et les citoyennes obtiennent une note globale de D quant à leurs connaissances relatives à l'action climatique. (...). Fait paradoxal : les individus ne savent pas qu'ils ne comprennent pas, alors que seulement $6 \%$ affirment ne pas y comprendre grand-chose. 
L'importance d'augmenter le niveau de littératie climatique au sein de la population québécoise ne semble donc faire aucun doute.

Par ailleurs, «savoir » quels sont les comportements à changer ne suffit pas. Il faut aussi «vouloir » et " pouvoir » les changer. C'est pourquoi le Front commun a invité une équipe de chercheuses en sciences de l'environnement, en psychologie comportementale, en communication climatique et en santé durable à mailler leurs connaissances scientifiques aux savoirs expérientiels d'organismes membres et de citoyen.ne.s-expert.e.s afin de coconstruire des outils et des approches d'accompagnement efficaces pour les personnes qui voudront adopter des modes de vie ZéN.

\section{«Chantiers ZéN en milieu de travail » (en développement)}

7 Le lieu de travail est un important milieu de vie. Les travailleuses et travailleurs s'y sentent concerné.e.s et interpellé.e.s. Elles et ils sont souvent les mieux placé.e.s pour connaître les défis d'une transition juste et les solutions à mettre en place. La mise sur pied de Chantiers ZéN comme espace de mobilisation, de formation, de planification et de mise en œuvre de mesures pour la transition des milieux de travail vers la carboneutralité s'impose donc comme une avenue importante à explorer, d'autant plus que la plupart des syndicats du Québec, étant membres du Front commun pour la transition énergétique, pourraient assumer le leadership d'une bonne partie de ces Chantiers ZéN.

\section{Des constats préliminaires}

Sans présumer des impacts du projet Québec ZéN à moyen et à long terme, il est déjà possible de faire un certain nombre de constats.

\section{La théorie du changement est mobilisatrice.}

Le besoin de visualiser la société post-carbone semble bien réel et le mot « espoir » a été prononcé à de nombreuses reprises après une première lecture de la Feuille de route. La confiance étant souvent contagieuse, tout comme son contraire, c'est même peut-être l'enthousiasme que la vision a suscité d'emblée qui a permis au projet de voir le jour.

31 Sur le plan quantitatif, il semble indéniable que la théorie du changement que nous avons adoptée a suscité une vaste adhésion. L'engagement assidu d'un grand nombre de membres du Front commun dans la coconstruction de la Feuille de route en est un indice, tout comme la participation d'un nombre substantiel d'allié.e.s externes. Entre juin 2019, à la veille de l'adoption du projet par l'Assemblée générale, et novembre 2021, le membership du Front commun est passé de 60 organisations représentant 900000 personnes à près de 100 organisations représentant 1,8 million de personnes. Il va sans dire qu'une croissance aussi rapide expose aussi le Front commun à une certaine fragilisation.

Il est pertinent d'engager les acteurs des milieux concernés dans la coconstruction du changement. 
Sans échapper complètement à toute critique, la Feuille de route et le projet Collectivités ZéN ne semblent pas avoir fait l'objet d'attaque majeure et ont au contraire déclenché de nombreuses offres de collaboration. Plusieurs de ces offres ont émané d'organisations membres du Front commun, mais d'autres sont venues de l'extérieur, notamment du milieu universitaire. Les témoignages mènent à penser qu'une approche axée sur la coconstruction du savoir et des solutions suscite l'adhésion de deux manières différentes: d'abord, en donnant aux participant.e.s l'occasion de faire leur marque et de s'approprier la démarche ; ensuite, et de manière peut-être plus significative, en brisant l'isolement et en cocréant ainsi l'espoir de voir se réaliser un projet collectif de transformation socio-écologique.

\section{Il n'est pas facile de ne laisser personne derrière.}

Le Front commun a placé au cœur de ses principes et de son action l'inclusion des groupes vulnérabilisés, marginalisés, victimes d'exclusion et de discrimination, notamment les peuples autochtones. L'un des critères adoptés pour une transition énergétique porteuse de justice sociale stipule ceci : «Une attention particulière doit être portée à ce que les mesures de transition énergétique soient émancipatrices, notamment en incluant les communautés dans les processus de façon transversale. ${ }^{11}$ " Cependant, sans surprise, l'expérience a confirmé qu'en cette matière, les bonnes intentions ne suffisent pas. Comment inclure les absent.e.s? Quelles pratiques faut-il mettre en place pour faire fleurir la confiance? Malgré les efforts et une volonté sincère, les réponses à ces questions commencent à peine à émerger, deux ans après le lancement officiel du projet. Les timides progrès enregistrés permettent toutefois d'espérer que l'inclusion cessera éventuellement d'être un objectif pour devenir une réalité.

6 Un cadre de gestion horizontal et distribué nourrit la mobilisation.

Par choix, mais aussi par nécessité, le Front commun a acquis au fil du projet Québec ZéN certaines expertises en gestion d'une coalition apprenante, en leadership partagé, en coconstruction de contenus et en innovation sociale. Loin d'être complets et sans doute appelés à demeurer constamment perfectibles, ces acquis semblent avoir grandement contribué à nourrir la mobilisation. Ils sont le fruit des efforts de longue haleine qui ont été faits et qui se poursuivent pour construire un mouvement social puissant, en transformant graduellement ce qui était au départ une coalition d'organisations disparates, dont les missions et intérêts s'entrechoquent à certains égards, en un écosystème performant au service de la transition socio-écologique.

Enfin, de façon transversale, nous observons l'importance de la collaboration avec le milieu universitaire pour mettre davantage la recherche au service du travail militant sur le terrain. Résolument axé sur le croisement de l'action et de la connaissance, le projet Québec ZéN a déjà donné lieu à de riches collaborations entre le Front commun pour la transition énergétique et le milieu de la recherche, plus spécifiquement dans le domaine de l'éducation relative à l'environnement. Ainsi, les résultats du projet FORJE (FORmation collaborative pour la Justice Énergétique) du Centre de recherche en éducation relative à l'environnement et à l'écocitoyenneté de l'UQAM (Brière et coll., 2021) ont nourri les réflexions d'un grand nombre de militant.e.s sur des questions et enjeux prioritaires soulevés dans le cadre du projet Québec ZéN. Le Front commun pour la transition énergétique espère vivement que les collaborations de ce type pourront se multiplier, notamment dans le cadre de projets de recherche-action participative. 


\section{BIBLIOGRAPHIE}

Brière, L., Moreau, G., Chatelain, M., Marleau, M.-È., Orellana, I., Prud'homme, M. et Riverin, J-A. (2021). Encourager et nourrir la formation réciproque au cour d'initiatives citoyennes de résistance et de transition énergétique. Rapport de recherche. Projet FORJE : FORmation collaborative pour la Justice Énergétique. Montréal : Les Publications du Centr'ERE.

Champagne St-Arnaud, V., Alexandre, M., Ducharme, M.-È., Lalloz, C., Poitras, P. et Daignault, P. (2020). Baromètre de l'action climatique 2020 : disposition des Québécois et des Québécoises envers les défis climatiques. Québec : Rapport du Laboratoire de l'action climatique (Université Laval) en collaboration avec « Unpointcinq ». Consulté sur https://static.lpcdn.ca/fichiers/html/ 4452/9186D_Barometre_2020_F.PDF

Khun, T.S. (1970), La Structure des révolutions scientifiques. Paris : Flammarion, collection. "Champs », $2^{\mathrm{e}}$ édition

Projet Québec ZéN (2020). Urgence climatique : Feuille de route

pour la transition du Québec vers la carboneutralité. Front commun pour la transition énergétique. Consulté sur www.pourlatransitionenergetique.org/feuille-de-route-quebec-zen/

Sauvé, L. (2018). Mémoires de luttes au creux des territoires. Relations, Printemps 2018, p. 25-26. Consulté sur https://www.erudit.org/fr/revues/rel/2018-n796-rel03644/88125ac.pdf

Sauvé, L. et Batellier, P. (2011-2012). La mobilisation citoyenne sur la question du gaz de schiste au Québec : Une exigence de démocratie. Nouveaux Cahiers du Socialisme, 6, «Écosocialisme ou barbarie » (sous la direction de Brouillette, V., Guay, N., Levy, A., Martin, E. et Poulin, R.), automne 2011, 224-236. Consulté sur www.mondequibouge.be/wp-content/images/2012/04/ Sauve-et-Batellier-Une-exigence-de-democratie.pdf

\section{NOTES}

1. www.pourlatransitionenergetique.org/

2. Ce positionnement s'exprime dans les critères du Front commun pour une transition énergétique porteuse de justice sociale: www.pourlatransitionenergetique.org/les-criteresdune-transition-energetique-porteuse-de-justice-sociale

3. Mission et vision : www.pourlatransitionenergetique.org/mission-vision/

4. www.pourlatransitionenergetique.org/feuille-de-route-quebec-zen/

5. À l'avant-plan des recherches ayant produit ces connaissances se trouvent les milliers de scientifiques $\mathrm{du}$ monde entier qui contribuent aux travaux du Groupe d'experts intergouvernemental sur l'évolution du climat (le GIEC), un organisme international créé en 1988 en vue de fournir des évaluations détaillées de l'état des connaissances scientifiques, techniques et socio-économiques sur les changements climatiques, leurs causes, leurs répercussions potentielles et les stratégies de parade.

6. Voir, à titre d'exemples, les travaux de l'Organisation mondiale de la santé (OMS) sur les impacts du réchauffement climatique sur la santé, ceux d'économistes comme Thomas Piketty sur le réchauffement climatique et les inégalités, les nombreuses recherches scientifiques établissant des liens entre le réchauffement climatique et l'effondrement de la biodiversité, les études sur le climat adoptant une perspective écoféministe. 
7. Voir, à titre d'exemples, les sites des ministères de l'Environnement du Québec et du Canada, du Ministère de l'Environnement de la France, d'ONG environnementales comme Équiterre ou la Fondation David Suzuki.

8. À titre d'exemples, le 28 septembre 2019, une foule estimée à un demi-million de personnes a marché à Montréal dans le cadre d'une "grève mondiale pour le climat »; en octobre 2019, 149 organisations, y compris un grand nombre d'organismes environnementaux et de groupes écologistes, ont soumis 187 mémoires dans le cadre des consultations publiques pour l'élaboration du Plan pour une économie verte 2030 du Québec.

9. Dans le contexte spécifique de la crise climatique, cette hypothèse a été renforcée par les conclusions d'une vaste mission de consultation réalisée en France sur les freins à la transition énergétique. Bruno Duverger, député à l'Assemblée nationale française et rapporteur, résumait ainsi le message principal de son rapport : « Nous avons besoin d'une vision à long terme et d'un effort de planification pour avancer dans la bonne direction. Aujourd'hui, nous appréhendons la transition énergétique par le petit bout de la lorgnette en multipliant les petites mesures, qui finissent par s'entrechoquer parce qu'elles ne se rapportent à aucune vision d'ensemble.» (Compte rendu no 33 - final), Mission d'information relative aux freins à la transition énergétique, 24 juin 2019. http://www.assemblee-nationale.fr/15/cr-transener/18-19/ c1819033.asp).

10. Comme l'ont observé entre autres Brière et coll. (2021), Sauvé (2018) et Sauvé et Batellier (2011-2012)

11. https://www.pourlatransitionenergetique.org/les-criteres-dune-transition-energetiqueporteuse-de-justice-sociale/

\section{RÉSUMÉS}

L'histoire environnementale du Québec met en évidence le rôle majeur des acteurs de la société civile pour repérer, exiger et opérer les changements majeurs qui s'imposent. En particulier, l'examen des mobilisations au fil des dernières décennies montre l'importance de la coconstruction des savoirs, du dialogue social et de l'expérience de l'action concertée pour favoriser le développement d'un pouvoir citoyen permettant de contribuer à la transformation des réalités socio-écologiques. S'inscrivant dans une telle dynamique, cet article présente les fondements et le cheminement d'un mouvement social d'envergure qui depuis quelques années, s'est saisi de la question climatique.

The environmental history of Quebec highlights the major role of civil society actors in identifying, demanding and implementing the major changes that are needed. In particular, an examination of mobilizations over the last few decades shows the importance of the coconstruction of knowledge, social dialogue and the experience of concerted action to promote the development of citizen power to contribute to the transformation of socio-ecological realities. In the context of this dynamic, this article presents the foundations and the progress of a large-scale social movement which, for several years now, has taken up the climate issue. 
INDEX

Mots-clés : transition énergétique, changements climatiques, Québec ZéN

Keywords : energy transition, climate change, Quebec ZeN

Index géographique: Québec

\section{AUTEUR}

\section{CAROLE DUPUIS}

Carole Dupuis est l'une des représentantes du mouvement citoyen au sein du comité de coordination du Front commun pour la transition énergétique depuis la création de cette coalition en 2015. Elle a cofondé le Mouvement écocitoyen « UNEplanète », un regroupement pan-québécois, et le groupe local Saint-Antoine-de-Tilly - Milieu de vie, qui a joué un rôle actif dans la lutte contre les forages au Québec. Avant de militer à temps plein à compter de 2014, elle a fait carrière en communication et en planification stratégique. 